\title{
A Narrative Review of Statin-Induced Rhabdomyolysis: Molecular Mechanism, Risk Factors, and Management
}

\author{
Nisa Safitri ${ }^{\prime}$ \\ Maya Fadila Alaina' \\ Dian Ayu Eka Pitalokal,2 \\ Rizky Abdulah (D) ${ }^{1,2}$ \\ 'Department of Pharmacology and \\ Clinical Pharmacy, Faculty of Pharmacy, \\ Universitas Padjadjaran, Sumedang, \\ 45363, Indonesia; ${ }^{2}$ Center of Excellence \\ in Higher Education for Pharmaceutical \\ Care Innovation, Universitas Padjadjaran, \\ Sumedang, 45363, Indonesia
}

\begin{abstract}
Although statins are effective for treating hypercholesterolemia, they can have various side effects, including rhabdomyolysis, a potentially fatal condition. This review evaluated the incidence and underlying molecular mechanism of statin-induced rhabdomyolysis and analyzed its risk factors, prevention, and management. We focused on the clinical and randomized clinical trials of statin monotherapies and combinations with other drugs. The primary mechanism of statin therapy-induced rhabdomyolysis is believed to be a decrease in ubiquinone (coenzyme Q) produced by the HMG-CoA pathway. Additionally, different types of lipophilic and hydrophilic statins play a role in causing rhabdomyolysis. Although statin-induced rhabdomyolysis has a low incidence, there is no guarantee that patients will be free of this side effect. Rhabdomyolysis can be prevented by reducing the risk factors, such as using CYP3A4 inhibitors, using high-dose statins, and strenuous physical activities.
\end{abstract}

Keywords: statin, hypercholesterolemia, adverse effect, rhabdomyolysis, risk factor

\section{Introduction}

Cholesterol, a fatty substance necessary for the proper functioning of the body, is critical for the synthesis of hormones and vitamin D. Cholesterol is transported through the blood by a specific class of particles called lipoproteins. Low-density lipoproteins (LDLs) carry liver cholesterol to the cells, and high-density lipoproteins (HDLs) remove excess cholesterol from different tissues and transport it back to the liver for elimination. ${ }^{1}$ LDL, also known as bad cholesterol, is the most important factor in cardiovascular disease development. LDL can cause atherosclerosis, the formation of plaque on the artery walls that reduces the blood flow, and can even cause a heart attack. ${ }^{2}$ The maximum limit of tolerated cholesterol is $200 \mathrm{mg} / \mathrm{dL}$ for total cholesterol and $100 \mathrm{mg} / \mathrm{dL}$ for LDL cholesterol (LDL-C), and the minimum limit for HDL cholesterol (HDL-C) is $50 \mathrm{mg} / \mathrm{dL}$. When the corresponding plasma cholesterol levels in a patient exceed these limits, the patient is defined as having a condition called hypercholesterolemia. ${ }^{3}$

Statins are well-known lipid-lowering agents and one of the world's most prescribed drugs. Statins lower cholesterol levels through three interconnected mechanisms. The first mechanism is the selective and competitive inhibition of 3-hydroxy-3-methylglutaryl-coenzyme A (HMG-CoA) reductase that limits the conversion speed of HMG-CoA to mevalonic acid, a precursor of sterols, including
Correspondence: Dian Ayu Eka Pitalok Tel +62 22-84288812

Email dian.pitaloka@unpad.ac.id 


\section{Graphical Abstract}

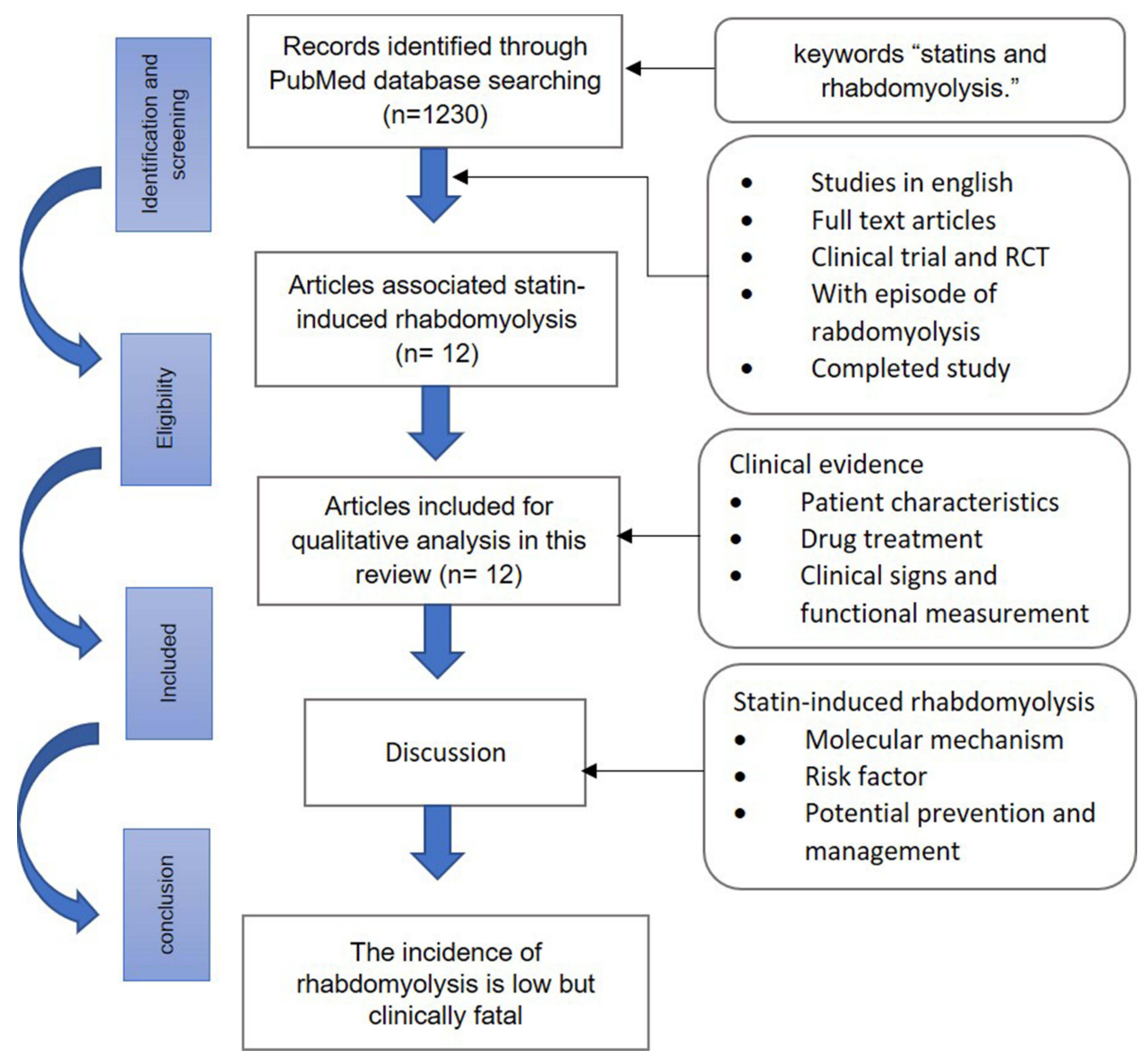

cholesterol in the mevalonate pathway, causing the substrate to bind to the active site and reducing cholesterol synthesis. ${ }^{4}$ The second indirect mechanism is to increase the receptor-mediated absorption of LDL, thereby reducing the level of plasma LDL. The third mechanism involves lowering the levels of LDL precursors, very-lowdensity and intermediate-density lipoproteins, to reduce the level of plasma LDL-C further. ${ }^{5}$

However, statins have been reported to exert side effects on muscles; the most common side effects are muscle pain, stiffness, myalgia, myopathy, and rhabdomyolysis. ${ }^{6}$ Rhabdomyolysis is caused by the damage to skeletal muscles that disrupts muscle integrity and releases muscle components, such as Creatine Kinase (CK), myoglobin, lactate dehydrogenase, aldolase, and electrolytes, into the bloodstream. ${ }^{7,8}$ Clinical presentations of rhabdomyolysis include muscle pain, swelling, weakness, and red urine because of increased myoglobin levels. ${ }^{9}$ Several reports have shown that the use of statins causes rhabdomyolysis. ${ }^{10-13}$ Although rhabdomyolysis rarely occurs, it remains a concern because of its severe clinical effects.

This review aims to study the evidence related to statin-induced rhabdomyolysis, identify the risk factors, and describe the potential prevention and management strategies. Additionally, we focus on the evidence reported in clinical trials and randomized controlled trials (RCTs) on statin monotherapies and statin-related multidrug therapies.

\section{Methods}

The selection for the related article published in 20012021 was conducted in the PubMed database using the keywords "statins and rhabdomyolysis." The inclusion criteria were English articles on the RCTs and clinical trials on statin-associated monotherapies or multidrug 


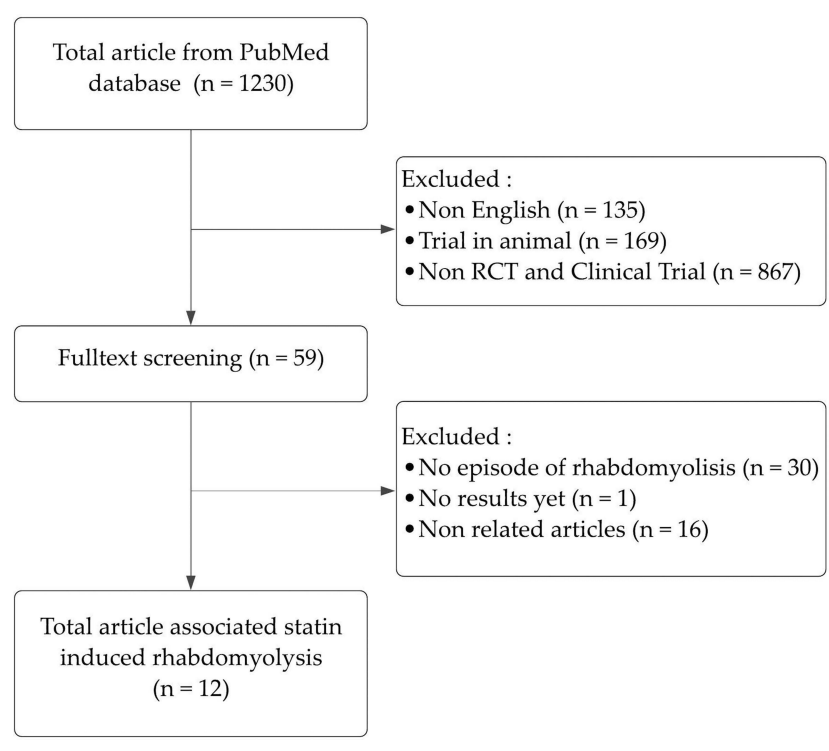

Figure I Flowchart of the literature search process.

therapies. A total of 1230 publications were found, of which 59 met the inclusion criteria. In addition, through full-text screening, we excluded the articles that did not describe an episode of rhabdomyolysis, study rhabdomyolysis, or have related research results. Of these 59 papers, only 12 were on the incidence of rhabdomyolysis because of statins: 7 were RCTs, 4 were clinical trials, and 1 was a Phase 1 clinical trial (Figure 1).

\section{Current Evidence of Statin-Induced Rhabdomyolysis Patient Characteristics}

The total number of patients reported in 12 studies was $1,129,477$, of which $102(0.009 \%)$ had statin-induced rhabdomyolysis (Table 1). The mean age of the patients was 63.57. Additionally, most of these patients were male (57.4\%). However, other characteristics, such as the body mass index and comorbidities, were not mentioned in all 12 studies.

\section{Drug Treatment}

Although the types and doses of statins used in the trials varied, simvastatin was the most broadly used type. For example, two studies reported using simvastatin as monotherapy. ${ }^{14,15}$ Abraldes et al administered simvastatin at $20 \mathrm{mg} /$ day for the first 15 days and continued with $40 \mathrm{mg} /$ day as the standard therapy to increase the survival rate of patients with cirrhosis. ${ }^{14}$ Meanwhile, Wierzbicki et al compared the effects of 80 and $120 \mathrm{mg} /$ day simvastatin on patients with hypercholesterolemia. ${ }^{15}$ Both studies showed simvastatin monotherapy to have a very strong effect in inducing rhabdomyolysis, as evidenced in the percentage of its occurrence (Table 1).

Meanwhile, three studies studied simvastatin in combination with other drugs. The study by HPS2-THRIVE reported a 4-week regimen of simvastatin ( $40 \mathrm{mg} /$ day), niacin ( $1 \mathrm{~g} /$ day), and laropiprant $(20 \mathrm{mg} /$ day $)$, followed by $3-6$ weeks of extended-release niacin ( $2 \mathrm{~g}$ /day) and laropiprant $(40 \mathrm{mg} /$ day). During the study, $10 \mathrm{mg}$ /day ezetimibe was given when the total cholesterol was less than $3.5 \mathrm{mmol} / \mathrm{L}^{13}$ This combination therapy-induced rhabdomyolysis at the same rate as the placebo group (simvastatin $40 \mathrm{mg} /$ day), at $0.109 \%$ and $0.114 \%$, respectively. Moreover, another study compared the efficacy of simvastatin monotherapy $(40 \mathrm{mg} /$ day $)$ in reducing LDL-C with that of a combination of simvastatin and ezetimibe $(10 \mathrm{mg} /$ day $) .{ }^{11}$ Furthermore, Pose et al compared the efficacy of two different doses of simvastatin (40 and $20 \mathrm{mg}$ / day) in combination with rifaximin ( $1200 \mathrm{mg} /$ day) and demonstrated that $40 \mathrm{mg} /$ day simvastatin induced rhabdomyolysis more than $20 \mathrm{mg} /$ day simvastatin. ${ }^{16}$

Conversely, Pedersen et al compared the efficacy of two types of statins in two doses: $20 \mathrm{mg} /$ day simvastatin and $40 \mathrm{mg} /$ day high-dose atorvastatin. ${ }^{17}$ The study showed that simvastatin at a lower dose had a greater effect on rhabdomyolysis, that is, by $0.07 \%$, than at a higher dose. ${ }^{17}$ The effectiveness of $80 \mathrm{mg} /$ day atorvastatin before and $24 \mathrm{~h}$ after percutaneous coronary intervention followed by $40 \mathrm{mg} /$ day atorvastatin for 30 days was already conducted in the RCT study. A total of three cases of rhabdomyolysis were reported in this study. ${ }^{10}$ Additionally, clinical trials were conducted to assess the safety of rosuvastatin (5-40 mg/day) in 16,874 patients ${ }^{18}$ and its side effects on skeletal muscles after fluvastatin and rosuvastatin use (dosages are unavailable). One case of rhabdomyolysis occurred in a patient who received rosuvastatin in combination with gemfibrozil. ${ }^{19}$ Furthermore, a phase 1 clinical trial study was conducted to evaluate the safety of rosuvastatin (1-8 $\mathrm{mg} / \mathrm{kg} /$ day $)$ and erlotinib $(150 \mathrm{mg} /$ day $)$ in treating advanced solid malignancies; one case of rhabdomyolysis was reported. ${ }^{12}$

Lastly, other studies did not list the type or dose of statins used. Enger et al conducted an RCT study to analyze the side effects of lipid-lowering agents, such as statins and fibrates. ${ }^{20}$ Based on the results, the percentage of statin-induced rhabdomyolysis increased when a statin was combined with fibrates; additionally, there was a correlation between the use of fibrates and rhabdomyolysis. Meanwhile, another RCT study reported the incidence of rhabdomyolysis in patients taking lipidlowering agents, including statins, but the dose of each drug 
Table I Studies on Statins and Rhabdomyolysis Included in This Review

\begin{tabular}{|c|c|c|c|c|}
\hline References & Study Design & $\begin{array}{l}\text { Number of } \\
\text { Patients }\end{array}$ & Treatment & Rhabdomyolysis \\
\hline \multirow[t]{2}{*}{ Pedersen et al ${ }^{17}$} & \multirow[t]{2}{*}{$\mathrm{RCT}$} & \multirow[t]{2}{*}{8888} & Simvastatin (20 mg/day) & $0.07 \%$ \\
\hline & & & Atorvastatin $(80 \mathrm{mg} /$ day $)$ & $0.05 \%$ \\
\hline \multirow[t]{3}{*}{ Enger et $\mathrm{al}^{20}$} & \multirow[t]{3}{*}{ RCT } & \multirow[t]{3}{*}{584,784} & Statin** & $0.003 \%$ \\
\hline & & & Statin** + Fenofibrate* & $0.015 \%$ \\
\hline & & & Statin $* *+$ Gemfibrozil ${ }^{*}$ & $0.207 \%$ \\
\hline \multirow[t]{2}{*}{ HPS2-THRIVE ${ }^{13}$} & \multirow[t]{2}{*}{ RCT } & \multirow[t]{2}{*}{25,673} & $\begin{array}{l}\text { Simvastatin }(40 \mathrm{mg} / \text { day })+\text { ER niacin }(2 \mathrm{~g} / \text { day }) \text { and laropiprant } \\
(40 \mathrm{mg} / \text { day })\end{array}$ & $0.109 \%$ \\
\hline & & & Simvastatin (40 mg/day) & $0.114 \%$ \\
\hline \multirow[t]{7}{*}{ Cziraky et $\mathrm{al}^{2 \mathrm{I}}$} & \multirow[t]{7}{*}{ RCT } & \multirow[t]{7}{*}{473,343} & Atorvastatin* & $0.0057 \%$ \\
\hline & & & Pravastatin* & $0.010 \%$ \\
\hline & & & Simvastatin* & $0.0055 \%$ \\
\hline & & & Lovastatin* & $0.0038 \%$ \\
\hline & & & Fluvastatin* & $0.016 \%$ \\
\hline & & & Rosuvastatin* & $0.012 \%$ \\
\hline & & & Cerivastatin* & $0.085 \%$ \\
\hline Abraldes et $\mathrm{al}^{14}$ & $\mathrm{RCT}$ & 147 & $\begin{array}{l}\text { Simvastatin }(20 \mathrm{mg} / \text { day) (first } 15 \text { days) followed by simvastatin } \\
(40 \mathrm{mg} / \text { day) thereafter }\end{array}$ & $2.9 \%$ \\
\hline Giugliano et al ${ }^{1 /}$ & $\mathrm{RCT}$ & $|5,28|$ & Simvastatin $(40 \mathrm{mg})+$ ezetimibe $(10 \mathrm{mg} /$ day $)$ & $0.137 \%$ \\
\hline Berwanger et al ${ }^{10}$ & RCT & 4191 & $\begin{array}{l}\text { Loading doses of atorvastatin }(80 \mathrm{mg} / \text { day }) \text {, followed by } \\
\text { atorvastatin }(40 \mathrm{mg} / \text { day })\end{array}$ & $0.142 \%$ \\
\hline \multirow[t]{2}{*}{ Wierzbicki et al ${ }^{15}$} & \multirow[t]{2}{*}{ Clinical Trial } & \multirow[t]{2}{*}{22} & Simvastatin (80 mg/day) & $0 \%$ \\
\hline & & & Simvastatin (I 20 mg/day) & $4.5 \%$ \\
\hline Shepherd et al ${ }^{18}$ & Clinical Trial & 16,876 & Rosuvastatin (5-40 mg/day) & $0.047 \%$ \\
\hline \multirow[t]{2}{*}{ Drobny et al $^{19}$} & \multirow[t]{2}{*}{ Clinical Trial } & \multirow[t]{2}{*}{198} & Rosuvastatin* & \multirow[t]{2}{*}{$3.03 \%$} \\
\hline & & & Fluvastatin* & \\
\hline Goss et al ${ }^{12}$ & $\begin{array}{l}\text { Clinical Trial } \\
\text { phase I }\end{array}$ & 24 & Rosuvastatin $(\mathrm{I}-8 \mathrm{mg} / \mathrm{kg} /$ day $)+$ erlotinib $(150 \mathrm{mg} /$ day $)$ & $4.167 \%$ \\
\hline \multirow[t]{2}{*}{ Pose et $\mathrm{al}^{16}$} & \multirow{2}{*}{$\begin{array}{l}\text { Clinical trial } \\
\text { phase } 2\end{array}$} & \multirow[t]{2}{*}{50} & Simvastatin $(40 \mathrm{mg} /$ day $)+$ rifaximin $(1200 \mathrm{mg} /$ day $)$ & $19 \%$ \\
\hline & & & Simvastatin $(20 \mathrm{mg} /$ day $)+$ rifaximin $(1200 \mathrm{mg} /$ day $)$ & $0 \%$ \\
\hline
\end{tabular}

Notes: *Dose is not available; **Dose and type of drug are not available.

Abbreviation: RCT, randomized controlled trial.

was not clearly stated. ${ }^{21}$ These findings suggest that simvastatin induces rhabdomyolysis at a higher rate than other types of statins, consistent with the theory that patients taking lipophilic statins have a higher risk of side effects and that increasing statin dose causes a higher ratio of side effects. ${ }^{22}$
Clinical Signs and Functional Measurement According to most studies, the patients with induced rhabdomyolysis experienced muscle weakness, myalgia, and fatigue. Additionally, they had CK levels above normal at more than $2000 \mathrm{U} / \mathrm{L}$ or 10 times the upper limit of normal (ULN). ${ }^{5,12,19}$ 
Moreover, their urine samples change to a darker color. ${ }^{19}$ However, some of the studies did not specifically list the symptoms experienced by patients and the laboratory test results.

Goss et al reported a rhabdomyolysis-related death. Of the 24 patients in the study, one experienced rhabdomyolysis after the administration of rosuvastatin and erlotinib and died. ${ }^{12}$ On day 6 (erlotinib only), this patient showed normal alanine transaminase, albumin, hepatic, renal, and muscle functions. However, on day 28 (rosuvastatin + erlotinib), the patient's CK level increased to more than $2000 \mathrm{U} / \mathrm{L}$. These findings demonstrate that the percentage of rhabdomyolysis due to statin therapy is relatively low. However, the authors did not mention the possibility of rhabdomyolysis in certain patients.

\section{Molecular Mechanism of Statin-Induced Rhabdomyolysis}

Although the primary mechanism of rhabdomyolysis due to statin therapy (Figure 2) remains unknown, statins are believed to induce skeletal muscle necrosis, probably because of the decrease in ubiquinone. In addition to cholesterol, the HMG-CoA pathway also produces other essential molecules, such as ubiquinone (coenzyme Q). Ubiquinone, a component of the mitochondrial respiratory chain, acts as a mitochondrial electron transport facilitator. Thus, inhibiting the HMG-CoA pathway may inhibit ubiquinone production and disrupt cellular energy production, causing muscular cell death. ${ }^{23}$

The integrity of skeletal muscles; the presence of a catabolic state can lead to muscle atrophy. The pathway mediates protein turnover through several enzymes, one of which is protein ligase (atrogin-1), which increases after statin therapy and is associated with muscle loss. ${ }^{22}$

Different types of lipophilic and hydrophilic statins play a role in the occurrence of rhabdomyolysis. In vitro studies indicate that lipophilic statins have a more significant myopathic effect than hydrophilic statins. Rhabdomyolysis is a severe form of myopathy. Lipophilic statins, such as atorvastatin, simvastatin, and fluvastatin, increase cell disruption through apoptosis and proteolysis. These statins can easily pass through the lipid layer membrane by passive transport; thus, they have higher toxic effects. ${ }^{22}$ This review's findings are in line with this theory. The incidence of rhabdomyolysis is more common in patients taking atorvastatin and simvastatin. These findings are also consistent with the research by

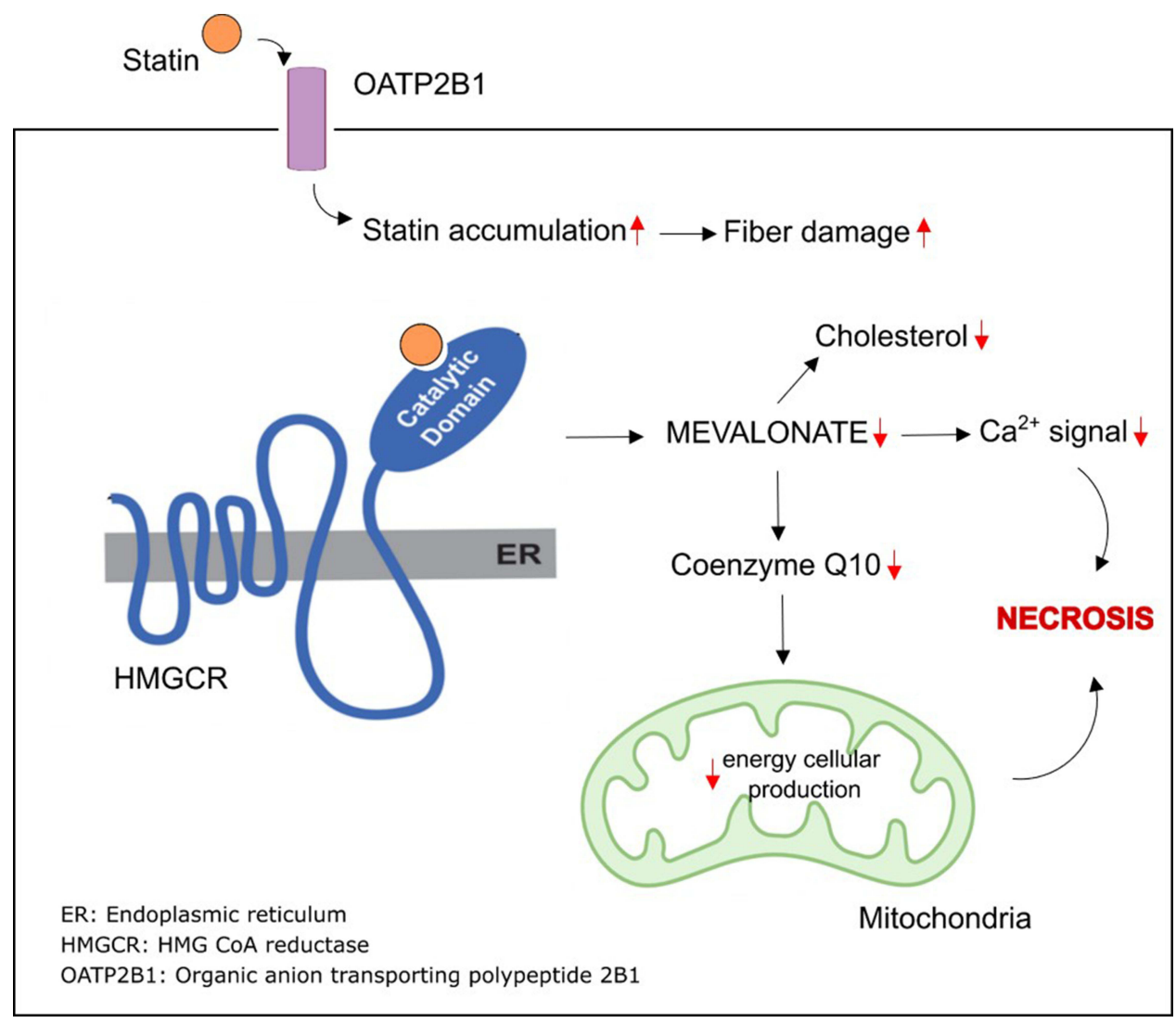

Figure 2 Potential mechanism underlying statin-induced rhabdomyolysis. 
Mendes et al, ${ }^{24}$ who examined the incidence of rhabdomyolysis due to statin therapy in the case reports published in 1990-2013 and found that simvastatin was the most widely reported statin associated with rhabdomyolysis (55 of 112 cases).

The effect of statins on myofibers has been observed in rats disease model. For example, statins can reduce intracellular adenosine triphosphate (ATP) and $\mathrm{Ca}^{2+}$ levels in the sarcoplasmic reticulum. It can also inhibit muscle contraction, causing contractile dysfunction and leading to cell damage, muscle cell death, and rhabdomyolysis. ${ }^{25,26}$

In the body, statins are also ionized into anions and get taken up by membrane transporters, such as the organic anion transporting polypeptide (OATP) transporter. ${ }^{27}$ Knauer et al reported that OATP2B1, an OATP isoform, facilitated the accumulation of statins in human myotubes and increased their myotoxicity. ${ }^{28}$ A similar result was reported by Tanaka et al who investigated the mechanism of statin myotoxicity in rats and found that OATP isoforms, OATP2b1 and OATP1a4, facilitated statin-induced myotoxicity and increased the risk of rhabdomyolysis. ${ }^{25}$

\section{Risk Factor Associated with Statin-Induced Rhabdomyolysis}

Statin-induced rhabdomyolysis may be due to statin monotherapies or combination therapies of statin and other drugs. However, certain drugs administered concurrently with statins increase the risk of rhabdomyolysis. Omar and Wilson examined the incidence of rhabdomyolysis due to statin therapy using the Adverse Event Reporting System of the Food and Drug Administration (FDA) and found that $50 \%$ of the statin-induced rhabdomyolysis cases are caused by drug-drug interactions. ${ }^{29}$

For example, combining statins with an inhibitor of the cytochrome P450 3A4 (CYP3A4) enzyme, such as verapamil, diltiazem, ritonavir, and amiodarone, potentially increases the risk of rhabdomyolysis. ${ }^{30-32}$ Since statins are oxidized by CYP3A4, which is produced by the liver, inhibiting CYP3A4 with certain drugs can reduce statin oxidation and decrease statins metabolism. Consequently, statins remain longer in the body, increasing the risk of side effects. ${ }^{24}$

Additionally, fibrates are associated with rhabdomyolysis. Fibrates can decrease LDL and increase HDL levels. However, Enger et $\mathrm{al}^{20}$ and another study have shown that fibrates, mostly fenofibrate, induce rhabdomyolysis. ${ }^{33}$ However, the mechanism underlying fibrate-induced rhabdomyolysis has not been precisely defined. ${ }^{34}$
The incidence of rhabdomyolysis may also increase with increasing statin dosage, as a higher dose elevates the plasma concentration of statin and its active metabolite. ${ }^{35}$ For example, a meta-analysis study by LaRosa $^{36}$ compared the effects of administering $10 \mathrm{mg}$ of atorvastatin to those of $80 \mathrm{mg}$. Subsequently, the author reported five cases of rhabdomyolysis at the higher dose and three cases at the lower dose.

Meanwhile, Schech et al identified the risk factors for rhabdomyolysis among statin users and found that statin users aged 65 years and older had four times the risk of hospitalization for rhabdomyolysis compared with those of the younger users; the increased risk was likely correlated with a decrease in organ function. ${ }^{37}$ The authors also observed a more than twofold increase in the risk of rhabdomyolysis among females.

Meanwhile, comorbidities, such as diabetes mellitus, were not found to be related to the risk. However, the association between rhabdomyolysis and renal diseases was statistically significant. Patients with pre-existing renal impairment taking cerivastatin may be at increased risk of rhabdomyolysis since the drug is excreted through the kidneys. ${ }^{37}$ Additionally, the association between the incidence of rhabdomyolysis with other diseases was reported in 147 patients with rhabdomyolysis incidence. Two of them had a history of liver disease. Liver disease is associated with the decreased expression of SLCO1B, which encodes an anion transporter that regulates statin absorption in the liver. Thus, mutations in SLCO1B may trigger statin-induced myopathy. ${ }^{14}$

Lastly, patients who are prescribed statins but engage in strenuous exercise also have an increased risk of rhabdomyolysis. One study showed an association between statin therapy and strenuous exercise. Patients given lovastatin had $62-77 \%$ higher CK levels after a workout than patients receiving a placebo. ${ }^{38}$ Additionally, another study using rodents showed that taking cerivastatin after using a treadmill could increase muscle damage. ${ }^{39}$ However, the association between the type of exercise and the severity of the risk of statin-induced rhabdomyolysis is unclear. Nevertheless, exercise can affect the absorption, distribution, metabolism, and excretion of statins, resulting in pharmacokinetic changes that can cause specific side effects. ${ }^{40}$ Strenuous exercise also causes excessive heat production, resulting in intracellular $\mathrm{Ca}^{2+}$ increase through ATP depletion. The loss of ATP disrupts the $\mathrm{Ca}^{2+}$-ATPase and $\mathrm{Na}^{+} / \mathrm{K}^{+}$-ATPase pumps, increasing intracellular $\mathrm{Ca}^{2+}$, activating proteases and reactive oxygen species, and 
finally causing muscle damage. ${ }^{41}$ In the PubMed database, there were 17 individual cases of heatstroke-associated rhabdomyolysis. Thus, it is recommended that individuals who work in hot environments anticipate rhabdomyolysis and take reasonable precautions. ${ }^{42}$

\section{Prevention and Management of Statin-Induced Rhabdomyolysis}

The best strategy to prevent statin-induced rhabdomyolysis is to lower all risk factors. Particularly, individuals who take statins and perform strenuous exercises, such as workers of certain occupations and athletes, should take frequent breaks to relax their muscles and relieve stress to decrease the risk of rhabdomyolysis. ${ }^{43}$ Additionally, clinicians who prescribe statins can also prioritize hydrophilictype statins such as pravastatin and rosuvastatin to reduce the potential side effects.

The FDA does not recommend prescribing simvastatin at high doses $(80 \mathrm{mg})$ because of the increased risk of developing statin-induced muscle injury. ${ }^{44}$ Simvastatin at $80 \mathrm{mg}$ can be prescribed if the patient exhibits no side effects after receiving the same therapy for 12 months. Additionally, the recommended dose of simvastatin is 5$40 \mathrm{mg}$ once daily. ${ }^{39}$ Therefore, the administration of less than $80 \mathrm{mg}$ of simvastatin or other types of statins is recommended. Although there is no guarantee that rhabdomyolysis or further muscle damage will not occur, lower dose of statin reduce the risk of muscle injury.

Additionally, since a combination of CYP3A4 inhibitors with statins can increase the risk of rhabdomyolysis, patients with hypercholesterolemia who have certain disease complications should avoid taking CYP3A4 inhibitors in combination with statins. ${ }^{45}$ Moreover, if a patient has rhabdomyolysis due to the administration of a lipophilic statin, eg, atorvastatin and simvastatin, switching to a hydrophilic statin, eg, pravastatin and rosuvastatin, may be reasonable.

Moreover, patients suspected of having rhabdomyolysis should undergo renal examination; then, statin therapy should be discontinued. Rhabdomyolysis is typically treated with intravenous rehydration or dialysis for patients with more severe symptoms. After assessing a patient's condition, statin can still be used at a lower dose. ${ }^{46}$ However, if the patient experiences increasing CK levels at up to five times the ULN, the statin regimen should be immediately discontinued, and the patient should be given another lipid-lowering drug. ${ }^{47}$
Lastly, Koba et al compared the efficacy of evolocumab with that of ezetimibe in patients with statin intolerance due to rhabdomyolysis. Evolocumab was more effective than ezetimibe in lowering LDL-C levels. After 12 weeks of drug administration, evolocumab decreased LDL-C levels by $59.5 \%$ of the baseline compared with ezetimibe by $20.3 \%{ }^{48}$ Thus, the use of evolocumab in a patient with statin intolerance should be recommended.

\section{Limitation}

However, our study has limitations. Although the selected articles in our study supported our study approach, we only used a single PubMed database to identify the articles that met our article selection criteria. Thus, there may be other related articles that have not been identified because they are not in PubMed. Despite the limitation, our study has described the potential risk factors for rhabdomyolysis; thus, our study can help reduce the risk of statininduced rhabdomyolysis.

\section{Conclusion}

Although statins offer benefits in managing hypercholesterolemia, they may cause rhabdomyolysis. The findings of this review suggest that although the incidence of statininduced rhabdomyolysis is low, its risk is still increasing because of the presence of triggering factors, such as the use of CYP3A4 inhibitors, initiation of high-dose statins, and strenuous physical activity. Therefore, the use of statins should be closely monitored, especially by measuring the CK levels that indicate rhabdomyolysis.

\section{Acknowledgments}

This study was funded by the Center of Excellence in Higher Education for Pharmaceutical Care Innovation Universitas Padjadjaran.

\section{Disclosure}

The authors report no conflicts of interest in this work.

\section{References}

1. Maxfield FR, van Meer G. Cholesterol, the central lipid of mammalian cells. Curr Opin Cell Biol. 2010;22(4):422-429. doi:10.1016/J.CEB.20 10.05.004

2. Ference BA, Ginsberg HN, Graham I, et al. Low-density lipoproteins cause atherosclerotic cardiovascular disease. 1. Evidence from genetic, epidemiologic, and clinical studies. A consensus statement from the European Atherosclerosis Society Consensus Panel. Eur Heart J. 2017;38(32):2459. doi:10.1093/EURHEARTJ/EHX144 
3. Grundy SM, Stone NJ, Bailey AL, et al. 2018 AHA/ACC/AACVPR/ AAPA/ABC/ACPM/ADA/AGS/APhA/ASPC/NLA/PCNA guideline on the management of blood cholesterol: a report of the American College of Cardiology/American Heart Association Task Force on clinical practice guidelines. Circulation. 2019;139(25):E1082-E1143. doi:10.1161/CIR.0000000000000625

4. Ward NC, Watts GF, Eckel RH. Statin toxicity. Circ Res. 2019;124 (2):328-350. doi:10.1161/CIRCRESAHA.118.312782

5. Stancu C, Sima A. Statins: mechanism of action and effects. J Cellullar Me. 2001;5(4):378-387. doi:10.1111/j.1582-4934.2001. tb00172.x

6. Stroes ES, Thompson PD, Corsini A, et al. Statin-associated muscle symptoms: impact on statin therapy-European Atherosclerosis Society consensus panel statement on assessment, aetiology and management. Eur Heart J. 2015;36:1012-1022. doi:10.1093/eurheartj/ehv043

7. Parker BA, Thompson PD. Effect of statins on skeletal muscle: exercise, myopathy, and muscle outcomes. Exerc Sport Sci Rev. 2012;40(4):188-194. doi:10.1097/JES.0b013e31826c169e

8. Torres PA, Helmstetter JA, Kaye AM, Kaye AD. Rhabdomyolysis: pathogenesis, diagnosis, and treatment. Ochsner J. 2015;15(1):58-69.

9. Zimmerman JL, Shen MC. Rhabdomyolysis. Chest. 2013;144 (3):1058-1065. doi:10.1378/chest.12-2016

10. Berwanger O, Santucci EV, de Silva PGM, et al. Effect of loading dose of atorvastatin prior to planned percutaneous coronary intervention on major adverse cardiovascular events in acute coronary syndrome: the SECURE-PCI randomized clinical trial. JAMA. 2018;319 (13):1331-1340. doi:10.1001/JAMA.2018.2444

11. Giugliano RP, Wiviott SD, Blazing MA, et al. Long-term safety and efficacy of achieving very low levels of low-density lipoprotein cholesterol: a prespecified analysis of the IMPROVE-IT trial. JAMA Cardiol. 2017;2(5):547-555. doi:10.1001/JAMACARDIO.2017.0083

12. Goss GD, Jonker DJ, Laurie SA, et al. A phase I study of high-dose rosuvastatin with standard dose erlotinib in patients with advanced solid malignancies. J Transl Med. 2016;14(1):1-11. doi:10.1186/ S12967-016-0836-6

13. Haynes R, Jiang L, Hopewell JC. HPS2-THRIVE randomized placebo-controlled trial in 25673 high-risk patients of ER niacin/ laropiprant: trial design, pre-specified muscle and liver outcomes, and reasons for stopping study treatment. Eur Heart J. 2013;34 (17):1279-1291. doi:10.1093/EURHEARTJ/EHT055

14. Abraldes JG, Villanueva C, Aracil C, et al. Addition of simvastatin to standard therapy for the prevention of variceal rebleeding does not reduce rebleeding but increases survival in patients with cirrhosis. Gastroenterology. 2016;150(5):1160-1170.e3. doi:10.1053/j. gastro.2016.01.004

15. Wierzbicki A, Lumb PJ, Chik G. Comparison of therapy with simvastatin $80 \mathrm{mg}$ and $120 \mathrm{mg}$ in patients with familial hypercholesterolaemia. Int J Clin Pract. 2001;55(10):673-675.

16. Pose E, Napoleone L, Amin A, et al. Safety of two different doses of simvastatin plus rifaximin in decompensated cirrhosis (LIVERHOPE-SAFETY): a randomised, double-blind, placebo-controlled, phase 2 trial. Lancet Gastroenterol Hepatol. 2020;5(1):31-41. doi:10.1016/S2468-1253(19)30320-6

17. Pedersen TR, Faergeman O, Kastelein JJP, et al. High-dose atorvastatin vs usual-dose simvastatin for secondary prevention after myocardial infarction: the IDEAL study: a randomized controlled trial. JAMA. 2005;294(19):2437-2445. doi:10.1001/JAMA.294.19.2437

18. Shepherd J, Vidt DG, Miller E, Harris S, Blasetto J. Safety of rosuvastatin: update on 16,876 rosuvastatin-treated patients in a multinational clinical trial program. Cardiology. 2007;107(4): 433-443. doi:10.1159/000100908

19. Drobný M, Pullmann R, Odalos I, Skerenova M, Sániova B. Incidence of skeletal muscle disorders after statins' treatment: consequences in clinical and EMG picture. Neuroendocrinol Lett. 2014;35(2):123-128.
20. Enger C, Gately R, Ming EE, Niemcryk SJ, Williams L, McAfee AT. Pharmacoepidemiology safety study of fibrate and statin concomitant therapy. Am J Cardiol. 2010;106(11):1594-1601. doi:10.1016/j. amjcard.2010.07.041

21. Cziraky MJ, Willey VJ, McKenney JM, et al. Risk of hospitalized rhabdomyolysis associated with lipid-lowering drugs in a real-world clinical setting. J Clin Lipidol. 2013;7(2):102-108. doi:10.1016/j. jacl.2012.06.006

22. Di Stasi SL, MacLeod TD, Winters JD, Binder-Macleod SA. Effects of statins on skeletal muscle: a perspective for physical therapists. Phys Ther. 2010;90(10):1530-1542. doi:10.2522/PTJ.20090251

23. Westwood FR, Bigley A, Randall K, Marsden AM, Scott RC. Statininduced muscle necrosis in the rat: distribution, development, and fibre selectivity. Toxicol Pathol. 2016;33(2):246-257. doi:10.1080/ 01926230590908213

24. Mendes P, Robles PG, Mathur S. Statin-induced rhabdomyolysis: a comprehensive review of case reports. Physiother Can. 2014;66 (2):124-132. doi:10.3138/ptc.2012-65

25. Tanaka S, Sakamoto K, Yamamoto M, et al. Mechanism of statin-induced contractile dysfunction in rat cultured skeletal myofibers. J Pharmacol Sci. 2010;114(4):454-463. doi:10.1254/ JPHS.10229FP

26. Sakamoto K, Honda T, Yokoya S, Waguri S, Kimura J. Rab-small GTPases are involved in fluvastatin and pravastatin-induced vacuolation in rat skeletal myofibers. FASEB J. 2007;21(14):4087-4094. doi:10.1096/FJ.07-8713COM

27. Shitara Y, Sugiyama Y. Pharmacokinetic and pharmacodynamic alterations of 3-hydroxy-3-methylglutaryl coenzyme A (HMG-CoA) reductase inhibitors: drug-drug interactions and interindividual differences in transporter and metabolic enzyme functions. Pharmacol Ther. 2006;112(1):71-105. doi:10.1016/J.PHARMTHERA.2006.03. 003

28. Knauer MJ, Urquhart BL, Schwabedissen HEM, et al. Human skeletal muscle drug transporters determine local exposure and toxicity of statins. Circ Res. 2010;106(2):297-306. doi:10.1161/CIRCRESAHA. 109.203596

29. Omar MA, Wilson JP. FDA adverse event reports on statin-associated rhabdomyolysis. Ann Pharmacother. 2002;36(2):288-295. doi:10. 1345/aph.1A289

30. Ezad S, Cheema H, Collins N. Statin-induced rhabdomyolysis: a complication of a commonly overlooked drug interaction. Oxf Med Case Rep. 2018;2018(3):86-88. doi:10.1093/OMCR/OMX104

31. Rowan CG, Brunelli SM, Munson J, et al. Clinical importance of the drug interaction between statins and CYP3A4 inhibitors: a retrospective cohort study in The Health Improvement Network. Pharmacoepidemiol Drug Saf. 2012;21(5):494-506. doi:10.1002/pds.3199

32. Yang BR, Seong JM, Choi NK, et al. Co-medication of statins with contraindicated drugs. PLoS One. 2015;10(5):1-11. doi:10.1371/journal.pone. 0125180

33. Danis R, Akbulut S, Ozmen S, Arikan S. Rhabdomyolysis-induced acute renal failure following fenofibrate therapy: a case report and literature review. Case Rep Med. 2010;2010:2-5. doi:10.1155/2010/ 537818

34. Wang D, Wang Y. Fenofibrate monotherapy-induced rhabdomyolysis in a patient with hypothyroidism. Med US. 2018;97(14):1-3. doi:10.1097/MD.000000000010318

35. Newman CB, Preiss D, Tobert JA, et al. Statin safety and associated adverse events a scientific statement from the American Heart Association. Arterioscler Thromb Vasc Biol. 2019;39(2):E38-E81. doi:10.1161/ATV.000000000000073

36. LaRosa JC, Grundy SM, Waters DD, et al. intensive lipid lowering with atorvastatin in patients with stable coronary disease. $N$ Engl $J$ Med. 2005;352(14):1425-1435. doi:10.1056/NEJMoa050461

37. Schech S, Graham D, Staffa J, et al. Risk factors for statin-associated rhabdomyolysis. Pharmacoepidemiol Drug Saf. 2007;16(3):352-358. doi:10.1002/pds. 1287 
38. Unnikrishnan D, Satish B. Exertion-induced rhabdomyolysis in a patient on statin therapy. Nephrol Dial Transplant. 2005;20 (1):244-247. doi:10.1093/ndt/gfh578

39. Seachrist JL, Loi CM, Evans MG, Criswell KA, Rothwell CE. Roles of exercise and pharmacokinetics in cerivastatin-induced skeletal muscle toxicity. Toxicol Sci. 2005;88(2):551-561. doi:10.1093/ toxsci/kfi305

40. Lenz TL, Lenz NJ, Faulkner MA. Potential interactions between exercise and drug therapy. Sports Med. 2004;34(5):293-306. doi:10.2165/00007256-200434050-00002

41. Tietze DC, Borchers J. exertional rhabdomyolysis in the athlete: a clinical review. Sports Health. 2014;6(4):336-339. doi:10.1177/ 1941738114523544

42. Yoshizawa T, Omori K, Takeuchi I, et al. Heat stroke with bimodal rhabdomyolysis: a case report and review of the literature. $J$ Intensive Care. 2016;4(1):1-5. doi:10.1186/s40560-016-0193-9

43. NIOSH. Prevention: rhabdomyolysis | NIOSH | CDC; 2019. Available from: https://www.cdc.gov/niosh/topics/rhabdo/prevention. html. Accessed July 14, 2021.
44. Food and Drug Administration. FDA drug safety communication: new restrictions, contraindications, and dose limitations for Zocor (simvastatin) to reduce the risk of muscle injury | FDA; 2021. Available fromhttps://www.fda.gov/drugs/drug-safety-and-availability/fda-drugsafety-communication-new-restrictions-contraindications-and-doselimitations-zocor. Accessed July 11, 2021.

45. Khalilieh S, Yee KL, Sanchez RI, et al. Results of a doravirine-atorvastatin drug-drug interaction study. Antimicrob Agents Chemother. 2017;61(2):e01364-16. doi:10.1128/AAC.01364-16

46. Barry AR, Beach JE, Pearson GJ. Prevention and management of statin adverse effects: a practical approach for pharmacists. Can Pharm J. 2018;151(3):179-188. doi:10.1177/1715163518768534

47. Ballantyne CM, Corsini A, Davidson MH, et al. Risk for myopathy with statin therapy in high-risk patients. Arch Intern Med. 2003;163 (5):553-564. doi:10.1001/ARCHINTE.163.5.553

48. Koba S, Inoue I, Cyrille M, et al. Evolocumab vs. ezetimibe in statin-intolerant hyperlipidemic Japanese patients: phase 3 GAUSS-4 trial. $J$ Atheroscler Thromb. 2020;27(5):471-484. doi:10. 5551/JAT.50963
Drug, Healthcare and Patient Safety

\section{Publish your work in this journal}

Drug, Healthcare and Patient Safety is an international, peer-reviewed open-access journal exploring patient safety issues in the healthcare continuum from diagnostic and screening interventions through to treatment, drug therapy and surgery. The journal is characterized by the rapid reporting of reviews, original research, clinical, epidemiological and post-marketing surveillance studies, risk management, health

\section{Dovepress}

literacy and educational programs across all areas of healthcare delivery. The manuscript management system is completely online and includes a very quick and fair peer-review system. Visit http://www.dovepress.com/testimonials.php to read real quotes from published authors. 